\title{
Quantum Neimark-Sacker bifurcation
}

\author{
I. I. Yusipov \& M. V. Ivanchenko*
}

Recently, it has been demonstrated that asymptotic states of open quantum system can undergo qualitative changes resembling pitchfork, saddle-node, and period doubling classical bifurcations. Here, making use of the periodically modulated open quantum dimer model, we report and investigate a quantum Neimark-Sacker bifurcation. Its classical counterpart is the birth of a torus (an invariant curve in the Poincaré section) due to instability of a limit cycle (fixed point of the Poincaré map). The quantum system exhibits a transition from unimodal to bagel shaped stroboscopic distributions, as for Husimi representation, as for observables. The spectral properties of Floquet map experience changes reminiscent of the classical case, a pair of complex conjugated eigenvalues approaching a unit circle. Quantum Monte-Carlo wave function unraveling of the Lindblad master equation yields dynamics of single trajectories on "quantumtorus" and allows for quantifying it by rotation number. The bifurcation is sensitive to the number of quantum particles that can also be regarded as a control parameter.

Bifurcation analysis, introduced by Poincare more than a century ago ${ }^{1}$, has become a primary approach in nonlinear dynamics and applications ${ }^{2,3}$. Its extensions to the quantum realm followed much later. The first and most celebrated example of bridging complex dynamics in the two domains is Hamiltonian chaos, which spectral signatures in quantum systems are profoundly understood by now ${ }^{4-7}$. For quite a while, the studies kept focused on Hamiltonian systems, seeking the footprints of bifurcations in the classical phase space on the properties of corresponding quantum equations and their solutions. The archetypal pitchfork and Hopf bifurcations ${ }^{2,3}$ in the mean-field equations were related to sharp changes of the ground-state entanglement in the corresponding quantum models ${ }^{8,9}$. A pitchfork bifurcation was also found to underpin the transition from Rabi to Josephson dynamics in experiments with rubidium Bose-Einstein condensate ${ }^{10}$.

Recent experimental advances in cavity quantum electrodynamics ${ }^{11}$, quantum optical systems ${ }^{12}$, Bose-Einstein condensates ${ }^{13-15}$, artificial atoms ${ }^{16}$, polaritonic devices ${ }^{17}$, and superconducting circuits ${ }^{18,19}$ have spurred attention to open quantum systems. These systems interact with their environments (or are subjected to actions from outside), and therefore their dynamics is essentially dissipative ${ }^{20,21}$. Although such systems evolve to a unique asymptotic state under broad conditions ${ }^{22,23}$, the dynamics has proved to be no less complex then the unitary one, framed by a set of eigenstates, yielding the states structurally and dynamically similar to classical chaotic attractors $^{24-30}$.

To date, several quantum counterparts of dissipative bifurcations have been described: pitchfork, saddle-node, and period doubling ${ }^{27-29,31-33}$. This is commonly done in the Markovian framework, and the dynamics of a model system is described with Lindblad equation ${ }^{21,23,34,35}$. The nonlinear mean-field equations used as a classical reference are obtained following Bogoliubov-Born-Green-Kirkwood-Yvon (BBGKY)-like approaches, by truncating the hierarchy of cumulants on the level of expectation values ${ }^{31}$ or keeping double correlators ${ }^{32,36}$. Routinely, quantum bifurcations are visualized by calculating quasi-classical phase space distributions, Husimi or Wigner-like ${ }^{37}$, which structural changes with a bifurcation parameter reproduce bifurcations in the classical phase space. For instance, the quantum period-doubling bifurcation is seen as the transition from unimodal to bimodal Husimi distribution ${ }^{27,33}$. At the same time, it has been demonstrated that quantum bifurcations can be observed directly as the structural changes in the asymptotic density matrix ${ }^{28}$. This approach allows to overcome the technical limitation of calculating Husimi distribution for the system size $N>10^{3}$, when it becomes computationally unfeasible. Both approaches, however, do not resolve dynamics on the quantum attractor.

In this paper we find and study the quantum Neimark-Sacker bifurcation, which classical counterpart is the birth of a torus (an invariant curve in the Poincaré/stroboscopic section) due to instability of a limit cycle (fixed point of the Poincaré map $)^{2}$. Exemplifying in the experimentally relevant open quantum periodically modulated dimer model, we show that the stroboscopic Husimi distribution exhibits a transition from the unimodal to 
bagel-shaped form - a section of "quantum torus" - for the boson interaction strength close to the bifurcation value for the mean-field model. Importantly, the same transformation is observed in the stroboscopic distribution of observables, obtained by the Monte Carlo wave-function stochastic unraveling of the Lindblad equation, the method, especially relevant in the context of quantum optics and cavity systems ${ }^{38-41}$. Dynamics of such individual quantum trajectories on "quntum torus" is suitably characterized by rotation number. Similar to the classical case, rotation numbers close to rational correspond to almost "periodic" multi-modal stroboscopic distributions. Finally, we demonstrate that the bifurcation is system size dependent, which plays a role of another bifurcation parameter.

The paper is organized as follows. In Section 1 we describe the quantum model, its nonlinear mean-field approximation, and numerical methods. Section 2 contains the main results. Section 3 gives a summary and outlook.

\section{Model and Methods}

Within the Markovian approximation framework (which assumes weak coupling to environment), the evolution of an open quantum system can be described by the Lindblad master equation ${ }^{21,23}$,

$$
\dot{\rho}=\mathscr{L}(\rho)=-i[H, \rho]+\mathscr{D}(\rho),
$$

where the first term in the r.h.s. captures the unitary evolution, and the second term describes the action of environment. We consider a system of $N$ indistinguishable interacting bosons, that hop between the sites of a periodically rocked dimer. This model is a popular theoretical testbed ${ }^{42-44}$, recently implemented in experiments ${ }^{45-47}$, known to exhibit regular and chaotic regimes ${ }^{27-29,33}$. Its unitary dynamics is governed by the Hamiltonian

$$
H(t)=-J\left(b_{1}^{\dagger} b_{2}+b_{2}^{\dagger} b_{1}\right)+\frac{2 U}{N} \sum_{g=1,2} n_{g}\left(n_{g}-1\right)+\varepsilon(t)\left(n_{2}-n_{1}\right) .
$$

Here, $J$ denotes the tunneling amplitude, $U$ is the interaction strength, and $\varepsilon(t)$ presents a periodical modulation of the on-site potentials. It is chosen as $\varepsilon(t)=\varepsilon(t+T)=A \sin (\Omega t), \Omega=2 \pi / T$, so that the amplitude $A$ is the dynamic energy offset between the two sites. Here $b_{j}$ and $b_{j}^{\dagger}$ are the annihilation and creation operators of a particle at site $j$, while $n_{j}=b_{j}^{\dagger} b_{j}$.

The system Hilbert space has dimension $N+1$ and can be spanned with $N+1$ Fock basis vectors, labeled by the number of bosons on the first site, $\{|n\rangle\}, n=0, \ldots, N$. Thus, the size of the model is controlled by the total number of bosons.

The dissipative term involves a single experimentally relevant jump operator ${ }^{48-50}$ :

$$
\begin{gathered}
\mathscr{D}(\rho)=\frac{\gamma}{N}\left(V \rho V^{\dagger}-\frac{1}{2}\left\{V^{\dagger} V, \rho\right\}\right), \\
V=\left(b_{1}^{\dagger}+b_{2}^{\dagger}\right)\left(b_{1}-b_{2}\right),
\end{gathered}
$$

which attempts to 'synchronize' the dynamics on the two sites by constantly recycling anti-symmetric out-phase modes into symmetric in-phase ones. The dissipative coupling constant $\gamma$ is taken to be time-independent.

Throughout the paper we set $J=1, \gamma=0.1, A=3.4, T=2 \pi$, and vary $U$ and $N$.

The computational analysis makes use of two methods to evolve the system numerically. First, we implement propagation of Eq. (1) by the fourth-order Runge-Kutta scheme. The evolution converges to a unique asymptotic solution, which in case of periodic modulation is a stable periodic trajectory (or a fixed point of the corresponding stroboscopic map, $\left.\mathscr{P}_{F}: \rho(m T) \rightarrow \rho((m+1) T), m=0,1,2, \ldots\right)$. For the particular system Eqs. (2) and (4), we set the integration time step of $5 \cdot 10^{-4} \mathrm{~T}$, and leave at least 100 modulation periods for transients to fade out.

The density matrix $\rho$ of the system with $N$ bosons can be visualized on the Bloch sphere by plotting the Husimi distribution $p(\vartheta, \phi)$, which can be obtained by projecting $\rho$ on the set of the generalized SU(2) coherent states ${ }^{51,52}$

$$
|\vartheta, \phi\rangle=\sum_{j=0}^{N} \sqrt{\left(\begin{array}{c}
N \\
j
\end{array}\right)}\left[\cos \frac{\vartheta}{2}\right]^{j}\left[e^{i \varphi} \sin \frac{\vartheta}{2}\right]^{N-j}|j\rangle .
$$

The corresponding nonlinear mean-field equations for the $(\theta, \varphi)$ phase variables are obtained by writing the master equation in terms of the spin operators $\mathscr{S}_{x}=\frac{1}{2 N}\left(b_{1}^{\dagger} b_{2}+b_{2}^{\dagger} b_{1}\right), \mathscr{S}_{y}=-\frac{i}{2 N}\left(b_{1}^{\dagger} b_{2}-b_{2}^{\dagger} b_{1}\right)$, $\mathscr{S}_{z}=\frac{1}{2 N}\left(n_{1}-n_{2}\right)$, and considering their evolution in the Heisenberg picture ${ }^{21,27,28}$. For a large number of $(N-1)$ bosons, the commutator $\left[\mathscr{S}_{x}, \mathscr{S}_{y}\right]=i \mathscr{S}_{z} \stackrel{N \rightarrow \infty}{=} 0$, vanishing as $\mathcal{O}\left(N^{-1}\right)$, as well as the similar ones for other cyclic permutations. Replacing operators with their expected values, $\mathscr{S}_{k}=\operatorname{tr}\left[\rho \mathscr{S}_{k}\right]$, and denote $\mathscr{S}_{k}$ by $S_{k}$, one can obtain

$$
\begin{aligned}
& \frac{d S_{x}}{d t}=2 \varepsilon(t) S_{y}-8 U S_{z} S_{y}+8 \gamma\left(S_{y}^{2}+S_{z}^{2}\right), \\
& \frac{d S_{y}}{d t}=-2 \varepsilon(t) S_{x}+8 U S_{x} S_{z}-2 J S_{z}+8 \gamma S_{x} S_{y}, \\
& \frac{d S_{z}}{d t}=-2 J S_{y}-8 \gamma S_{x} S_{z},
\end{aligned}
$$


where proportional to $\gamma$ terms of lower order in $N$ are neglected. The quantity $S^{2}=S_{x}^{2}+S_{y}^{2}+S_{z}^{2}$ is a constant of motion, so the mean-field evolution is restricted to the surface of a Bloch sphere, $\left(S_{x}, S_{y}, S_{z}\right)=\frac{1}{2}[\cos (\phi) \sin (\vartheta)$, $\sin (\phi) \sin (\vartheta), \cos (\vartheta)$ ], yielding the equations of motion

$$
\begin{aligned}
& \dot{\vartheta}=-2 J \sin (\phi)+4 \gamma \cos (\phi) \cos (\vartheta), \\
& \dot{\phi}=-2 J \frac{\cos (\vartheta)}{\sin (\vartheta)}-2 \varepsilon(t)+4 U \cos (\vartheta)-4 \gamma \frac{\sin (\phi)}{\sin (\vartheta)} .
\end{aligned}
$$

The corresponding particle number in the first site is then recovered as $n=\frac{N}{2}(1+\cos (\vartheta))$.

This nonlinear dynamical system plays a reference role in the bifurcation analysis further. In numerical experiments we record the consecutive stroboscopic values, $\{\vartheta(m T), \phi(m T)\}, m=0,1,2, \ldots$, and construct the histograms normalized to the largest value 1 for each parameter set, complemented by Poincaré sections for the stroboscopic map.

Second, we employ the Monte-Carlo wave function (also "quantum trajectory" or "quantum jump") method $^{38,39}$ to unravel Lindblad master Eq. (1) into an ensemble of quantum trajectories. It recasts the evolution of the model system into the ensemble of systems described by wave functions, $\psi_{r}(t), r=1,2, \ldots, M_{r}$, governed by an effective non-Hermitian Hamiltonian, $\tilde{H}$. This Hamiltonian incorporates the dissipative operator $V$, which is responsible for the decay of the norm,

$$
i \dot{\psi}=\tilde{H} \psi, \tilde{H}=H-\frac{i}{2} V^{\dagger} V .
$$

When the norm drops below a threshold, that is randomly chosen each time, a quantum jump is simulated, such that the wave function is transformed according to $\psi \rightarrow V \psi$ and then normalized ${ }^{20}$.

The density matrix can then be sampled from a set of $M_{r}$ realizations as $\rho\left(t_{\mathrm{p}} ; M_{\mathrm{r}}\right)=\frac{1}{M_{r}} \sum_{j=1}^{M_{\mathrm{r}}}\left|\psi_{j}\left(t_{\mathrm{p}}\right)\right\rangle\left\langle\psi_{j}\left(t_{\mathrm{p}}\right)\right|$, which, given an initial pure state $\psi^{\text {init }}$, converges towards the solution of Eq. (1) at time $t_{\mathrm{p}}$ for the initial density matrix $\rho^{\text {init }}=\left|\psi^{\text {init }}\right\rangle\left\langle\psi^{\text {init }}\right|$. We make use of the recently developed high-performance realization of the $\operatorname{method}^{53}$ and generate $M_{\mathrm{r}}=10^{2}$ different trajectories for averaging, leaving $t_{0}=2 \cdot 10^{3} \mathrm{~T}$ time for relaxation towards an asymptotic state, and following the dynamics for up to $t=10^{3} \mathrm{~T}$.

Thus we produce stroboscopic plots for the expectation values of the two observables, the number of particles on the left site of the dimer, $n(t)$, and the energy, $e(t)$,

$$
\begin{gathered}
n(t)=\left\langle\psi(t)\left|b_{1}^{\dagger} b_{1}\right| \psi(t)\right\rangle, \\
e(t)=\langle\psi(t)|H| \psi(t)\rangle .
\end{gathered}
$$

Quantum trajectories allow for an insight to dynamics in the asymptotic regime - on quantum attractor beyond the stationary Husimi projection picture.

\section{Results}

We first investigate the nonlinear mean-field equations, Eq. (7), treating interaction strength $U$ as a bifurcation parameter. There one observes the emergence of an invariant curve for a stroboscopic map $\mathscr{F}:\{\theta(m T), \phi(m T)\} \rightarrow$ $\{\theta((m+1) T), \phi((m+1) T)\}$ by the Neimark-Sacker bifurcation at $U \sim 0.11$ and its succession by period-6 cycle at $U \sim 0.18$ (Figs. 1(a) and 2). Further it develops into a chaotic attractor, that ultimately disappears through a crisis, when the stable fixed point is recovered.

We interrogate whether quantum equations manifest an analogue of the classical Neimark-Sacker bifurcation and study its properties.

A sufficiently large number of particles, $N=500$, should bring the system close to the classical limit. The one-parameter bifurcation diagram displays the probabilities to find a given number of particles on the left site of a dimer, taken at stroboscopic times $m T, m=0,1,2, \ldots$, numerically given by the diagonal elements of the density matrix, $\rho_{n, n}(m T)$. The qualitative structure of the bifurcation diagram obtained from the mean-field model is reproduced in the quantum case quite well, as for the birth of a torus, as for the onset of chaos and ultimate recovery of a stable fixed point, cf. Fig. 1(b). However, even for $N=500$ some details differ, like the quantum bifurcation within $U \in[0.6,0.7]$, that does not have a counterpart in the mean-field model. The discrepancy stems from truncation of double and higher order correlators, $\mathcal{O}\left(N^{-1}\right)$, in the mean-field approximation, retaining only expectation values, cf. Eqs. (6 and 7). We conjecture that by going to a higher-order mean-field approximation, one would obtain a higher-dimensional nonlinear system, where the corresponding classical bifurcation would emerge.

Next, we demonstrate the correspondence between the asymptotic states at stroboscopic Poincaré sections (for mean-field model) on the plane $\{\vartheta, \phi\}$ and Husimi distributions for the quantum system, Fig. 2. Indeed, the projection of the quantum attractor onto the classical phase space reveals the emergence of a bagel-shaped distribution after the invariant curve in the Poincaré section of the mean-field equations. Moreover, one observes the formation of a multi-period orbit on the quantum torus later on, cf. Fig. 2(d), the scenario typical of classical systems.

There are, however, quantitative differences: the bagel is already present at $U=0.1$ for the quantum model with $N=500$, cf. Fig. 2(a), while the mean-field model still has a fixed point; the size of the bagel is slightly greater than the invariant curve in the classical case, cf. Fig. 2(b,c); the formation of a periodic orbit in the quantum case occurs at lower $U$, cf. Fig. 2(d). 


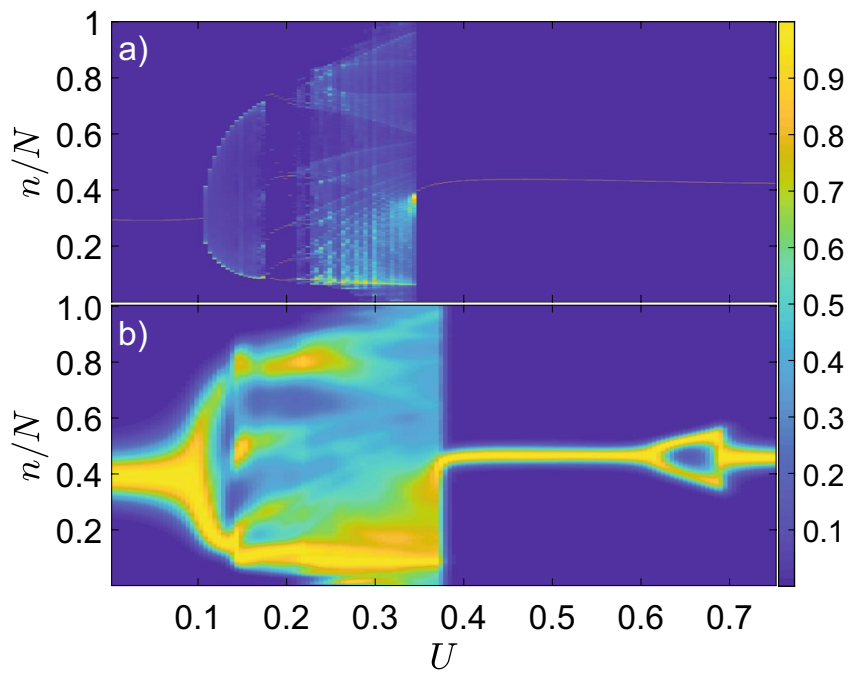

Figure 1. One-parameter bifurcation diagrams for the classical and quantum systems. Color codes the histogram for (a) classical and (b) quantum trajectories, the maximal element for each value of $U$ is normalized to $1, N=500$.

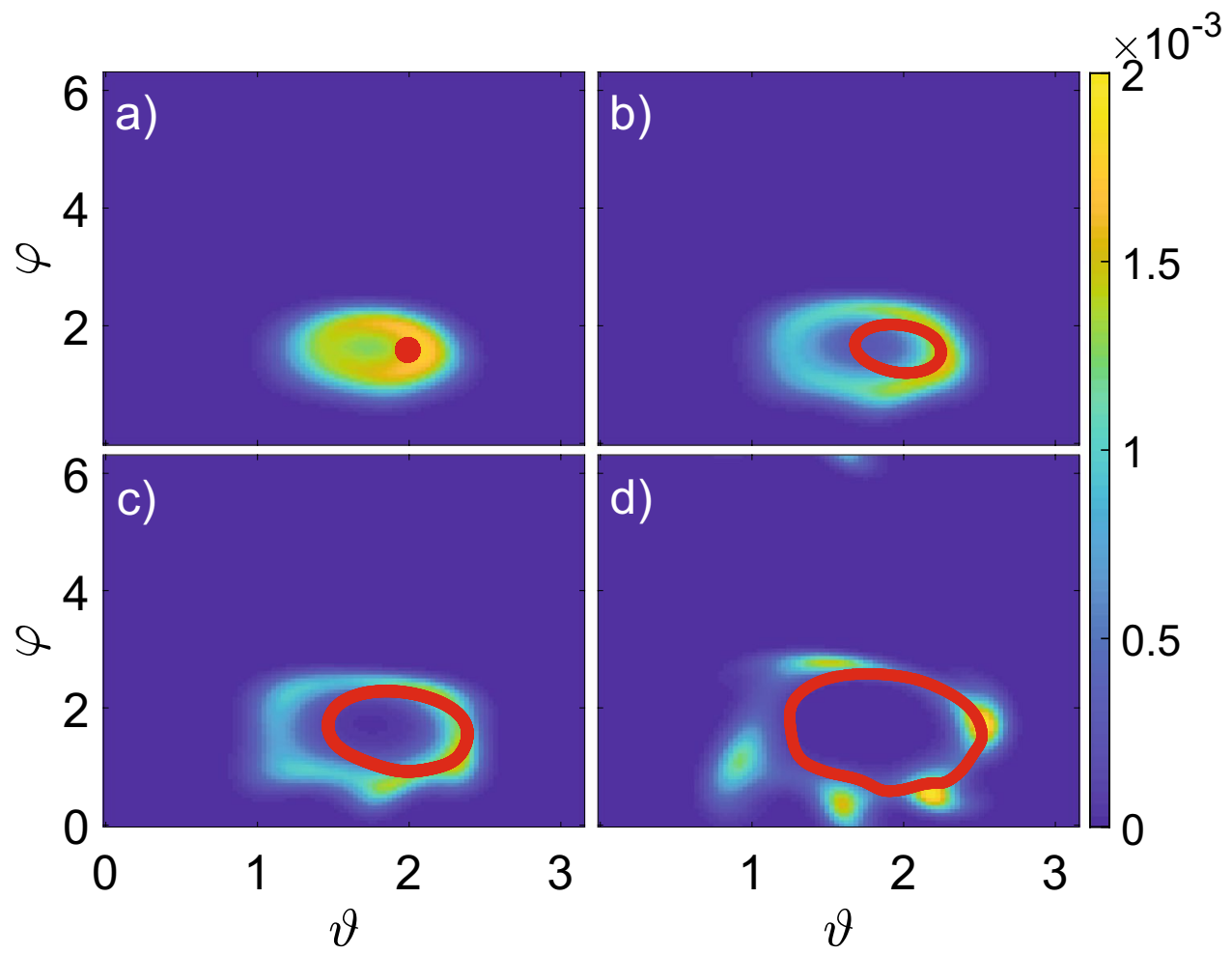

Figure 2. Stroboscopic Husimi distribution for the density matrix depending on interaction strength. Distributions are drawn for $(\mathbf{a}) U=0.1 ;(\mathbf{b}) U=0.1125 ;$ (c) $U=0.125 ;$ (d) $U=0.15$ and overlaid by Poincaré maps for the mean-field model (red). Here $N=500$.

We further investigated, whether quantum Neimark-Sacker bifurcation can be identified directly in quantum observables. Sampling attractor with quantum trajectories (cf. Section 1), we reconstruct histograms for stroboscopic observables $n(m T), e(m T)$, Eq. (10), and again witness the emergence of bagel-shaped distributions following quantum bifurcation, cf. Fig. 3 and compare to Fig. 2.

Quantum trajectory unraveling allows to get a deeper insight into the dynamics on quantum torus. That is, one can define the phase $\theta_{m}$ for each pair of stroboscopic observables, $n(m T), e(m T)$, as a polar angle, the origin placed at the center of mass of the stroboscopic $2 D$ histogram, which is normalized in both dimensions to the same interval $[-1,1]$ (see Fig. 4 , inset), and thus calculate the instantaneous rotation (or winding) number 


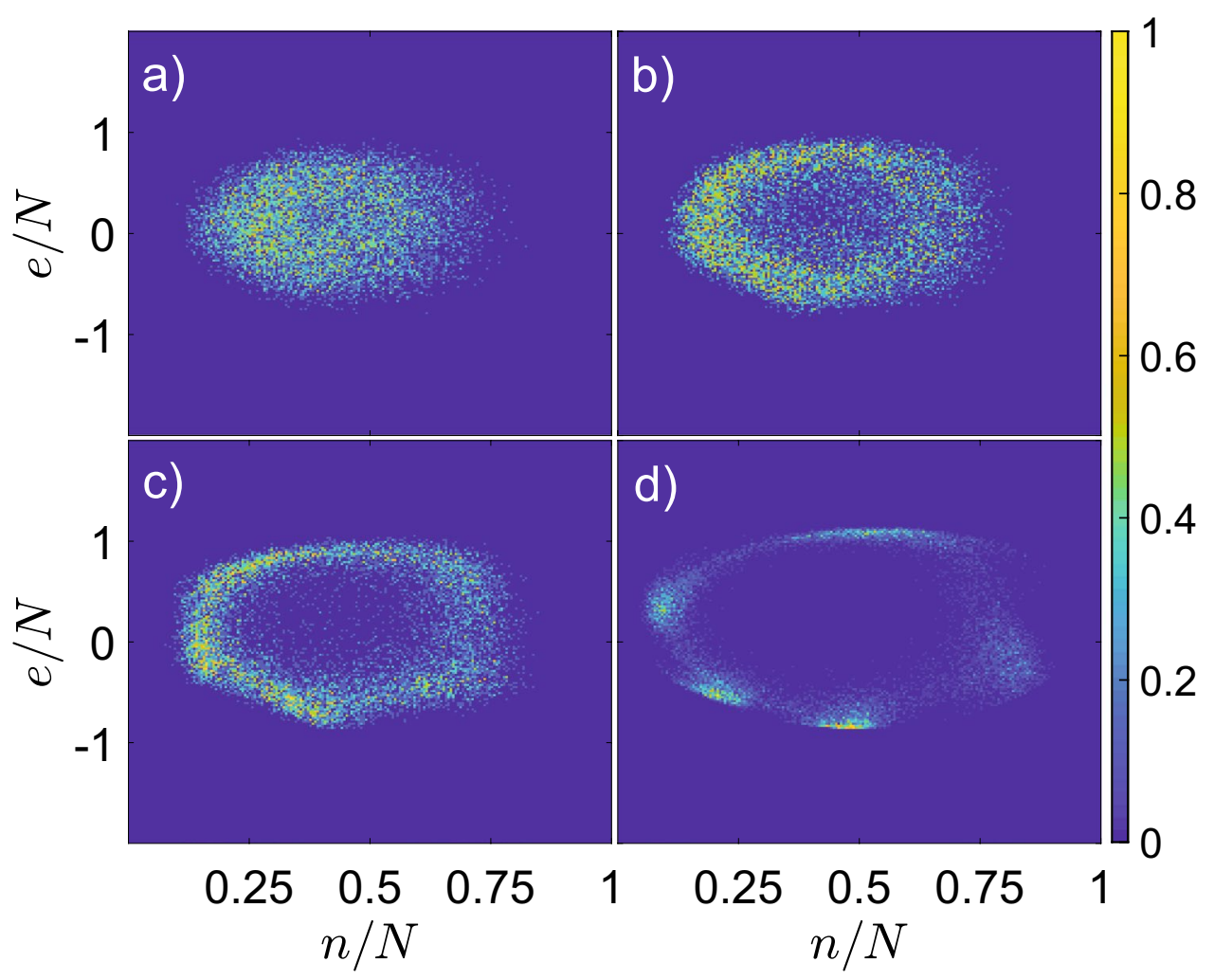

Figure 3. Histograms of expectation values for quantum trajectories at stroboscopic times. Trajectories are taken for different interaction strength: $(\mathbf{a}) U=0.1 ;(\mathbf{b}) U=0.1125 ;(\mathbf{c}) U=0.125 ;(\mathbf{d}) U=0.15$. The axes are scaled by the particle number, $n(m T) / N, e(m T) / N$, here $N=500$.

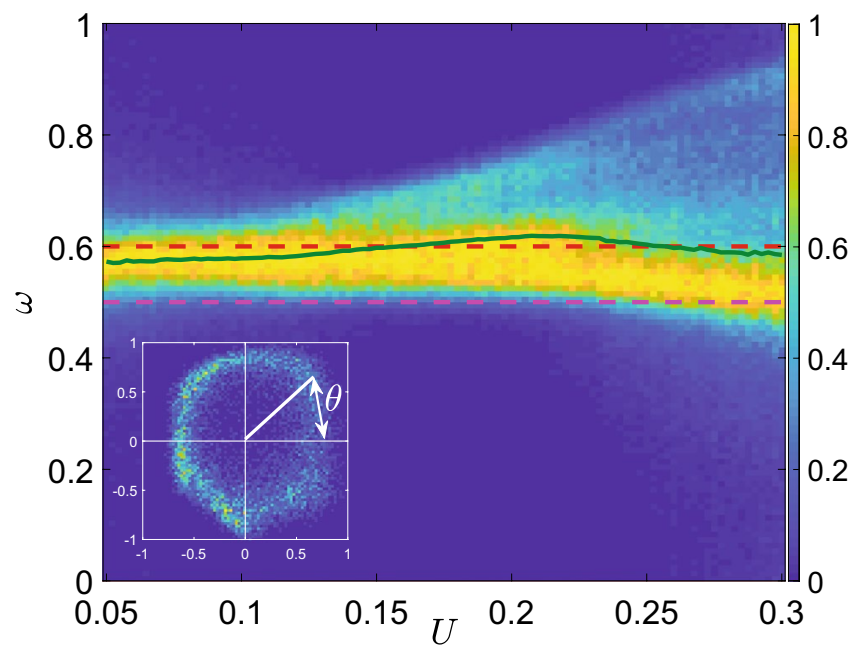

Figure 4. Color coded histogram of instantaneous rotation number, $\omega$, for quantum trajectories in dependence on interaction strength, $U$. The maximal element for each value of $U$ is normalized to 1 . Solid line corresponds to the average rotation number, dashed lines indicate the levels for rational $\omega=1 / 2$ and $\omega=3 / 5$. Here $N=250$. Inset: the phase $\theta$ is defined as a polar angle for centered and normalized observables.

$$
\omega_{m}=\frac{\theta_{m}-\theta_{m-1}}{2 \pi} \bmod 1
$$

In classical dynamics, the time-averaged $\omega$ discriminates two cases. For rational $\omega=p / q, p, q \in \mathcal{N}$, the invariant torus contains a stable period- $q$ orbit that is observed as an asymptotic solution, while for irrational $\omega$ the trajectories cover the torus densely. The regimes interchange with system parameters. 


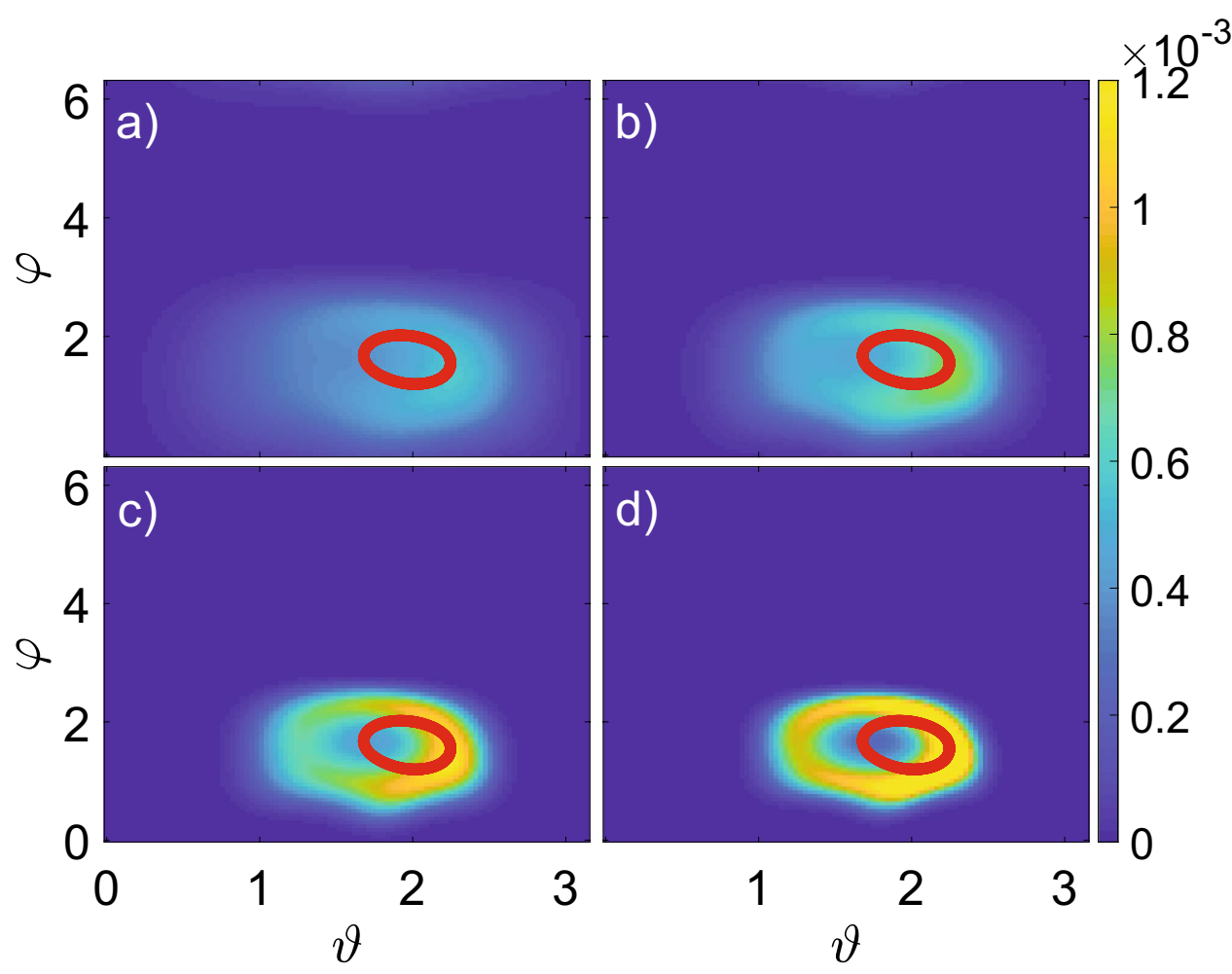

Figure 5. Diameter of a bagel, $D$, in the Husimi distribution in dependence on the interaction strength, $U$. Results are obtained for the different number of particles: $N=50$ (blue); $N=100$ (red); $N=250$ (green); $N=500$ (purple). Inset: eigenvalues of Floquet map, $\mathscr{P}_{F}$, on a complex plane about the quantum bifurcation point (blue) and a unit circle (orange), $U=0.12, N=50$; red points mark $\mu_{2,3}$.

The dynamics on quantum torus can be characterized by the probability distribution of $\omega_{m}$, Fig. 4 . About the bifurcation point $U^{*} \approx 0.1$ the distribution is well-localized about $\omega=0.58$, different from the period doubling value $1 / 2$, the bifurcation present for the other parameter values ${ }^{28,33}$. Our case is analogous to irrational rotation number for classical torus, so that quantum trajectories densely cover the bagel. The average rotation number increases with interaction strength, and becomes rational $\omega=3 / 5$ at $U \approx 0.15$. Then the stroboscopic distributions obtain a clear footprint of the corresponding period-5 structure (Figs. 2(c,d) and 3(c,d)), as in the classical case.

The bifurcation also affects the spectral properties of the system. This can be demonstrated by calculating and diagonalizing the Floquet map $\mathscr{P}_{F}=\mathscr{T} \exp \left[\int_{0}^{T} \mathscr{L} d t\right]$, where $\mathscr{T}$ is time-ordering operator, that describes evolution of the density operator over a period of modulation under Eq. (1) ${ }^{27}$. The largest eigenvalue of its spectrum, $\left\{\mu_{k}\right\}$, $k=1, \ldots,(N+1)^{2}$, is always unity, $\mu_{1}=1$, the rest are inside the unit circle, as per the dissipative nature of $\mathscr{L}$. We follow the next to largest eigenvalues and find a conjugated pair that approaches the unit circle at the point of quantum bifurcation, $\mu_{2,3} \approx e^{ \pm i \theta_{0}}$ (Fig. 5, inset). Its complex phases are consistent with the rotation number, $\theta_{0} \approx 2 \pi \omega$. Repeating the treatment of ref. ${ }^{33}$, one obtains that the two-time correlation of an observable in the asymptotic regime is incommensurate with the time-scale of modulation. Note that the spectral gap $1-\left|\mu_{2,3}\right|$ decreases with $N$, yielding the classical conjugate multipliers of the limit cycle at the Neimark-Sacker bifurcation point ${ }^{2}$ in the infinite-size limit, $N \rightarrow \infty$. It also gives an estimate of relaxation time from a random initial condition to the asymptotic state, $t \sim\left(1-\left|\mu_{23}\right|\right)^{-1}$, which can become very large with $N$, it follows.

Finally, we investigate the dependence on the number of bosons, $N$. The classical limit is formally obtained for $N \rightarrow \infty$, but the traits of classical Neimark-Sacker bifurcation are reproduced deep in the quantum regime, as soon as $N \sim 25$. In fact, the number of particles, $N$, can be considered as a bifurcation parameter itself. For instance, one can explore the case $U=0.1125$, when an invariant curve is already present in the mean-field model, cf. Figs. 2(b) and 3(b). However, the Husimi distribution for the quantum dimer with the relatively small number of particles, $N=50$, is still unimodal, cf. Fig. 6(a). Increasing $N$ one observes a transformation to a bagel shape (Fig. 6(b-d)), i.e. Neimark-Sacker bifurcation with the system size $N$.

In classical nonlinear systems the size of the invariant curve generically scales as a square root above the bifurcation point ${ }^{2}$. For the quantum bifurcation we define the diameter of the bagel $D$ in the Husimi distribution as the distance between two maxima in the section $\varphi=\pi / 2$. When the distribution is unimodal, we set $D=0$. The resulting curves $D(U)$ confirm a pronounced dependence on the number of particles, in particular, for the bifurcation point, cf. Fig. 5. The scaling of $D(U)$ about the bifurcation point also depends on the number of bosons and is not universal. 


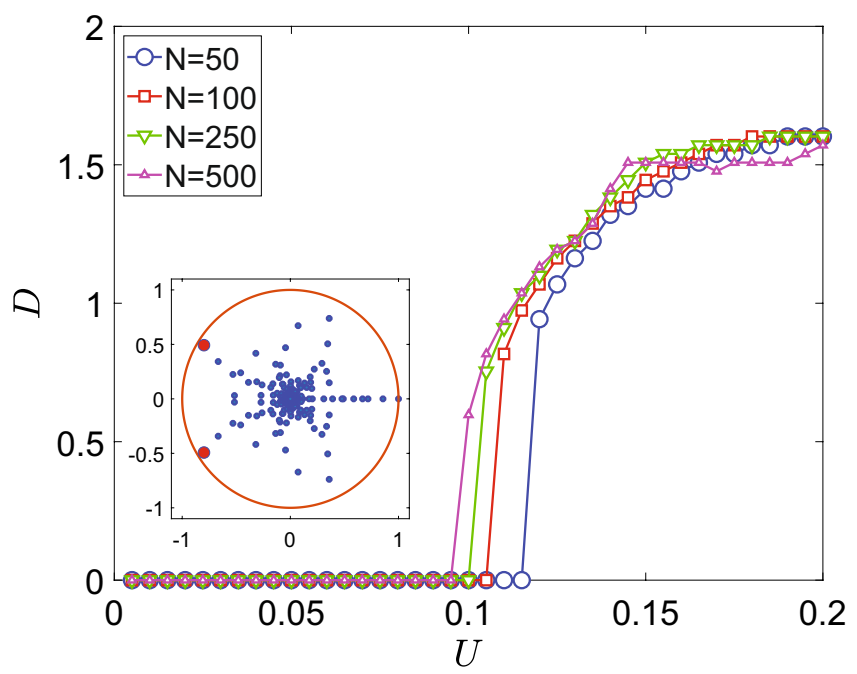

Figure 6. Stroboscopic Husimi distribution for the density matrix depending on the number of particles. Distributions are drawn for (a) $N=50$; (b) $N=100$; (c) $N=250 ;$ (d) $N=500$ and overlaid by the Poincaré map for the mean-field model (red). Here $U=0.1125$.

\section{Conclusions}

We found and investigated the quantum counterpart of the classical Neimark-Sacker bifurcation in an open periodically modulated quantum dimer. The classical bifurcation in a dissipative nonlinear system is a birth of torus from a limit cycle (or an invariant curve from a fixed point for a time-discrete map). Concurrently, a conjugate pair of complex Floquet multipliers of a periodic trajectory crosses the unit circle.

The quantum Neimark-Sacker bifurcation is manifested by emergence of bagel-shaped stroboscopic distributions, as for Husimi projection on the classical phase space, as for quantum observables. The bifurcation is also seen in the spectral properties of the corresponding Floquet map, as a pair of its conjugate eigenvalues approaching the unit circle. Quantum trajectories technique allows for unraveling the dynamics on the "quantum torus", and a suitably generalized rotation number on it. In analogy to the classical case, irrational and rational rotation numbers yield bagel (dense torus) and multi-modal (periodic orbit on torus) distributions. The quantum bifurcation depends on the system size - the number of bosons, and more sensitively for fewer particles, away from the mean-field limit. As such the number of particles is a bifurcation parameter.

We expect that the bifurcation can be found in the other open non-equilibrium quantum setups, to name a modulated open Dicke model, where its classical counterpart has been reported ${ }^{54}$. Thereby, cavity and circuit QED systems appear the first candidates for its experimental observation.

Received: 4 September 2019; Accepted: 1 November 2019;

Published online: 29 November 2019

\section{References}

1. Poincaré, H. Sur les courbes définies par les équations différentielles. American Journal of Mathematics 7, 203-258 (1885).

2. Kuznetsov, Y. A. Elements of applied bifurcation theory, vol. 112 (Springer Science \& Business Media, 2013).

3. Blanchard, P., Devaney, R. \& Hall, G. Differential Equations (Thomson Brooks/Cole, 1998).

4. Casati, G. \& Ford, J. Stochastic behavior in classical and quantum Hamiltonian systems. vol. 93 of Lecture Notes in Physics (SpringerVerlag, Berlin-New York, 1979).

5. Gutzwiller, M. C. Chaos in classical and quantum mechanics, vol. 1 of Interdisciplinary Applied Mathematics (Springer-Verlag, New York, 2013).

6. Haake, F., Wiedemann, H. \& Życzkowski, K. Lyapunov exponents from quantum dynamics. Annalen der Physik 504, 531-539 (1992).

7. Guhr, T., Müller-Groeling, A. \& Weidenmüller, H. A. Random-matrix theories in quantum physics: common concepts. Physics Reports 299, 189-425 (1998).

8. Hines, A. P., McKenzie, R. H. \& Milburn, G. J. Quantum entanglement and fixed-point bifurcations. Physical Review A 71, 042303 (2005).

9. Nemes, M. et al. Quantum entanglement and fixed point Hopf bifurcation. Physics Letters A 354, 60-66 (2006).

10. Zibold, T., Nicklas, E., Gross, C. \& Oberthaler, M. K. Classical bifurcation at the transition from Rabi to Josephson dynamics. Physical Review Letters 105, 204101 (2010).

11. Walther, H., Varcoe, B. T. H., Englert, B.-G. \& Becker, T. Cavity quantum electrodynamics. Reports on Progress in Physics 69, 1325-1382 (2006).

12. Aspelmeyer, M., Kippenberg, T. J. \& Marquardt, F. Cavity optomechanics. Reviews of Modern Physics 86, 1391-1452 (2014).

13. Liang, Z. X., Zhang, Z. D. \& Liu, W. M. Dynamics of a bright soliton in Bose-Einstein condensates with time-dependent atomic scattering length in an expulsive parabolic potential. Phys. Rev. Lett. 94, 050402 (2005).

14. Li, L., Li, Z., Malomed, B. A., Mihalache, D. \& Liu, W. M. Exact soliton solutions and nonlinear modulation instability in spinor Bose-Einstein condensates. Phys. Rev. A 72, 033611 (2005).

15. Wang, D.-S., Hu, X.-H., Hu, J. \& Liu, W. M. Quantized quasi-two-dimensional Bose-Einstein condensates with spatially modulated nonlinearity. Phys. Rev. A 81, 025604 (2010).

16. You, J. Q. \& Nori, F. Atomic physics and quantum optics using superconducting circuits. Nature 474, 589-597 (2011) 
17. Feurer, T. Spatiotemporal coherent control of lattice vibrational waves. Science 299, 374-377 (2003).

18. Barends, R. et al. Digital quantum simulation of fermionic models with a superconducting circuit. Nature Communications 6, 7654 (2015).

19. Roushan, P. et al. Spectroscopic signatures of localization with interacting photons in superconducting qubits. Science 358, 1175-1179 (2017).

20. Carmichael, H. An open systems approach to quantum optics: lectures presented at the Université Libre de Bruxelles, October 28 to November 4, 1991, vol. 18 (Springer Science \& Business Media, 2009).

21. Breuer, H.-P. \& Petruccione, F. The theory of open quantum systems (Oxford University Press, 2002).

22. Spohn, H. An algebraic condition for the approach to equilibrium of an open N-level system. Letters in Mathematical Physics 2, 33-38 (1977).

23. Alicki, R. \& Lendi, K. Quantum dynamical semigroups and applications, vol. 717 (Springer, 2007).

24. Spiller, T. P. \& Ralph, J. F. The emergence of chaos in an open quantum system. Physics Letters A 194, 235-240 (1994).

25. Brun, T. A., Percival, I. C. \& Schack, R. Quantum chaos in open systems: a quantum state diffusion analysis. Journal of Physics A: Mathematical and General 29, 2077-2030 (1996).

26. Carlo, G. G., Benenti, G., Casati, G. \& Shepelyansky, D. L. Quantum ratchets in dissipative chaotic systems. Physical Review Letters $94(2005)$.

27. Hartmann, M., Poletti, D., Ivanchenko, M., Denisov, S. \& Hänggi, P. Asymptotic floquet states of open quantum systems: the role of interaction. New Journal of Physics 19, 083011 (2017).

28. Ivanchenko, M. V. et al. Classical bifurcation diagrams by quantum means. Annalen der Physik 529, 1600402 (2017).

29. Carlo, G. G., Ermann, L., Rivas, A. M. F., Spina, M. E. \& Poletti, D. Classical counterparts of quantum attractors in generic dissipative systems. Physical Review E 95, 062202 (2017).

30. Yusipov, I. I., Vershinina, O. S., Denisov, S., Kuznetsov, S. P. \& Ivanchenko, M. V. Quantum Lyapunov exponents beyond continuous measurements. Chaos: An Interdisciplinary Journal of Nonlinear Science 29, 063130 (2019).

31. Meaney, C. P., Duty, T., McKenzie, R. H. \& Milburn, G. J. Jahn-Teller instability in dissipative quantum systems. Physical Review A 81, 043805 (2010).

32. Tomadin, A., Diehl, S. \& Zoller, P. Nonequilibrium phase diagram of a driven and dissipative many-body system. Physical Review A 83, $013611(2011)$

33. Wang, R. R. W., Xing, B., Carlo, G. G. \& Poletti, D. Period doubling in period-one steady states. Physical Review E 97, 020202 (2018).

34. Lindblad, G. On the generators of quantum dynamical semigroups. Communications in Mathematical Physics 48, 119-130 (1976).

35. Gorini, V. Completely positive dynamical semigroups of N-level systems. Journal of Mathematical Physics 17, 821-825 (1976).

36. Meiser, D. \& Holland, M. J. Intensity fluctuations in steady-state superradiance. Physical Review A 81, 063827 (2010).

37. Stockmann, H.-J. Quantum chaos (Cambridge University Press, 2007).

38. Dum, R., Parkins, A. S., Zoller, P. \& Gardiner, C. W. Monte Carlo simulation of master equations in quantum optics for vacuum, thermal, and squeezed reservoirs. Physical Review A 46, 4382-4396 (1992).

39. Mølmer, K., Castin, Y. \& Dalibard, J. Monte Carlo wave-function method in quantum optics. Journal of the Optical Society of America B 10, 524-538 (1993).

40. Plenio, M. B. \& Knight, P. L. The quantum-jump approach to dissipative dynamics in quantum optics. Reviews of Modern Physics 70, 101-144 (1998).

41. Daley, A. J. Quantum trajectories and open many-body quantum systems. Advances in Physics 63, 77-149 (2014).

42. Vardi, A. \& Anglin, J. R. Bose-Einstein condensates beyond mean field theory: Quantum backreaction as decoherence. Physical Review Letters 86, 568-571 (2001).

43. Trimborn, F., Witthaut, D. \& Wimberger, S. Mean-field dynamics of a two-mode Bose-Einstein condensate subject to noise and dissipation. Journal of Physics B: Atomic, Molecular and Optical Physics 41, 171001 (2008).

44. Poletti, D., Bernier, J.-S., Georges, A. \& Kollath, C. Interaction-induced impeding of decoherence and anomalous diffusion. Physical Review Letters 109, 045302 (2012).

45. Weiss, C. \& Teichmann, N. Differences between mean-field dynamics and N-particle quantum dynamics as a signature of entanglement. Physical Review Letters 100, 140408 (2008).

46. Gross, C., Zibold, T., Nicklas, E., Estève, J. \& Oberthaler, M. K. Nonlinear atom interferometer surpasses classical precision limit. Nature 464, 1165-1169 (2010).

47. Tomkovič, J. et al. Experimental observation of the Poincaré-Birkhoff scenario in a driven many-body quantum system. Physical Review A 95, 011602 (2017).

48. Diehl, S. et al. Quantum states and phases in driven open quantum systems with cold atoms. Nature Physics 4, 878-883 (2008).

49. Kraus, B. et al. Preparation of entangled states by quantum Markov processes. Physical Review A 78, 042307 (2008).

50. Schindler, P. et al. Quantum simulation of dynamical maps with trapped ions. Nature Physics 9, 361-367 (2013).

51. Arecchi, F. T., Courtens, E., Gilmore, R. \& Thomas, H. Atomic coherent states in quantum optics. Physical Review A 6, 2211-2237 (1972).

52. Perelomov, A. Generalized coherent states and their applications. Springer, Texts and Monographs in Physics 6, 156-164 (1986).

53. Volokitin, V. et al. Computation of the asymptotic states of modulated open quantum systems with a numerically exact realization of the quantum trajectory method. Physical Review E 96, 053313 (2017).

54. Gong, Z., Hamazaki, R. \& Ueda, M. Discrete time-crystalline order in cavity and circuit QED systems. Physical Review Letters 120, 040404 (2018).

\section{Acknowledgements}

The authors acknowledge support of the President of Russian Federation grant No. MD-6653.2018.2, Basis

Foundation grant No. 17-12-279-1, and Russian Foundation for Basic Research grant No. 18-32-20221.

\section{Author contributions}

I.I.Y. and M.V.I. contributed equally to conducting research, preparing and reviewing the manuscript.

\section{Competing interests}

The authors declare no competing interests.

\section{Additional information}

Correspondence and requests for materials should be addressed to M.V.I.

Reprints and permissions information is available at www.nature.com/reprints.

Publisher's note Springer Nature remains neutral with regard to jurisdictional claims in published maps and institutional affiliations. 
(c) (i) Open Access This article is licensed under a Creative Commons Attribution 4.0 International License, which permits use, sharing, adaptation, distribution and reproduction in any medium or format, as long as you give appropriate credit to the original author(s) and the source, provide a link to the Creative Commons license, and indicate if changes were made. The images or other third party material in this article are included in the article's Creative Commons license, unless indicated otherwise in a credit line to the material. If material is not included in the article's Creative Commons license and your intended use is not permitted by statutory regulation or exceeds the permitted use, you will need to obtain permission directly from the copyright holder. To view a copy of this license, visit http://creativecommons.org/licenses/by/4.0/.

(C) The Author(s) 2019 